\title{
Observational evidence for composite grains in an AGB outflow
}

\section{MgS in the extreme carbon star LL Pegasi}

\author{
R. Lombaert ${ }^{1}$, B. L. de Vries ${ }^{1}$, A. de $\operatorname{Koter}^{2,3}$, L. Decin ${ }^{1,2}$, M. Min ${ }^{2,3}$, K. Smolders ${ }^{1}$, \\ H. Mutschke ${ }^{4}$, and L. B. F. M. Waters 2,5
}

\author{
${ }^{1}$ Instituut voor Sterrenkunde, KU Leuven, Celestijnenlaan 200D 2401, 3001 Leuven, Belgium \\ e-mail: robin.lombaert@ster. kuleuven.be \\ 2 Astronomical Institute "Anton Pannekoek", University of Amsterdam, PO Box 94249, 1090 GE Amsterdam, The Netherlands \\ 3 Astronomical Institute Utrecht, University Utrecht, PO Box 80000, 3508 TA Utrecht, The Netherlands \\ 4 Astrophysikalisches Institut und Universitäts-Sternwarte, Schillergäßchen 2-3, 07745 Jena, Germany \\ 5 Netherlands Institute for Space Research, Sorbonnelaan 2, 3584 CA Utrecht, The Netherlands \\ Received 8 June 2012 / Accepted 1 July 2012
}

\section{ABSTRACT}

\begin{abstract}
The broad $30 \mu \mathrm{m}$ feature in carbon stars is commonly attributed to $\mathrm{MgS}$ dust particles. However, reproducing the $30 \mu \mathrm{m}$ feature with homogeneous $\mathrm{MgS}$ grains would require much more sulfur relative to the solar abundance. Direct gas-phase condensation of $\mathrm{MgS}$ occurs at a low efficiency. Precipitation of $\mathrm{MgS}$ on $\mathrm{SiC}$ precursor grains provides a more efficient formation mechanism, such that the assumption of homogeneous $\mathrm{MgS}$ grains may not be correct. Using a Monte Carlo-based radiative transfer code, we aim to model the $30 \mu \mathrm{m}$ feature of the extreme carbon star LL Peg with MgS dust particles. We find that for LL Peg this modeling is insensitive to the unknown $\mathrm{MgS}$ optical properties at $\lambda<10 \mu \mathrm{m}$. When $\mathrm{MgS}$ is allowed to be in thermal contact with amorphous carbon and $\mathrm{SiC}$, the amount of $\mathrm{MgS}$ required to reproduce the strength of $30 \mu \mathrm{m}$ feature agrees with the solar abundance of sulfur, thereby resolving the reported MgS mass problem. We conclude that $\mathrm{MgS}$ is a valid candidate to be the carrier of the $30 \mu \mathrm{m}$ feature when it is part of a composite grain population that has optical properties representative of an ensemble of particle shapes.
\end{abstract}

Key words. stars: AGB and post-AGB - stars: abundances - stars: evolution - stars: mass-loss - stars: winds, outflows - stars: carbon

\section{Introduction}

The broad $30 \mu \mathrm{m}$ feature in the thermal continuum emission of carbon stars was identified in 1985 by Goebel \& Moseley (1985) as due to magnesium sulfide (MgS). Begemann et al. (1994) presented optical data for $\mathrm{MgS}$ and compared them to the thermal continuum emission of $\mathrm{CW}$ Leo to confirm $\mathrm{MgS}$ as the likely carrier of the $30 \mu \mathrm{m}$ feature. The Infrared Space Observatory (ISO; Kessler et al. 1996) observed a large sample of carbon stars (e.g. Yamamura et al. 1998; Jiang et al. 1999; Szczerba et al. 1999; Hrivnak et al. 2000; Volk et al. 2002) displaying a diversity in strength, shape and width of the feature. In an analysis of this data set, Hony et al. (2002) concluded that the shape of the $30 \mu \mathrm{m}$ feature can be best reproduced when the grains are not perfect spheres. Zhang et al. (2009) modeled the $30 \mu \mathrm{m}$ feature in the proto-planetary nebula HD 56126 using pure MgS grains. Assuming the grains to be irradiated by unattenuated stellar light, their analysis needed the optical properties of $\mathrm{MgS}$ at wavelengths $\lambda<10 \mu \mathrm{m}$. Unfortunately, such measurements are lacking, as yet. Assuming relatively high absorption efficiencies in this regime, these authors required an amount of $\mathrm{MgS}$ up to ten times the amount of available atomic sulfur to explain the strength of the $30 \mu \mathrm{m}$ feature. Owing to this mass problem, Zhang and collaborators argued against $\mathrm{MgS}$ as the carrier of the feature.

In this letter, we report on the observational evidence for composite grains in the outflow of the high mass-loss rate asymptotic giant branch (AGB) star LL Peg, also known as AFGL 3068. We show that MgS is a viable candidate to explain the $30 \mu \mathrm{m}$ feature independent of the absorbing efficiencies of these grains at $\lambda<10 \mu \mathrm{m}$. Moreover, we show that if one assumes thermal contact between the dust species in the outflow, the mass problem in HD 56126 reported by Zhang et al. (2009) does not occur in LL Peg. Finally, we discuss the formation of composite grains in AGB outflows.

\section{Data}

The spectral energy distribution (SED) of LL Peg was constructed by combining spectra taken with the Short Wavelength Spectrometer (SWS) and Long Wavelength Spectrometer (LWS) on board ISO. The SWS data were retrieved from the Sloan et al. (2003) database; the LWS data from the ISO data archive. The LWS data were rescaled to the calibrated SWS data. We corrected for interstellar reddening following the extinction law of Chiar \& Tielens (2006), with an extinction correction factor in the $K$-band of $A_{\mathrm{K}}=0.01 \mathrm{mag}$ (Drimmel et al. 2003).

\section{Modeling the thermal energy distribution}

We combined two numerical codes to reproduce the ISO observations of LL Peg. The first is MCMax (Min et al. 2009), a dust continuum radiative transfer code based on a Monte Carlo method (Bjorkman \& Wood 2001), which computes the dust temperature self-consistently from the thermal energy balance equation. The second is GASTRoNOoM (Decin et al. 2006, 2010), which calculates the momentum transfer from dust to gas in the outflow. The results of these two modeling tools were iterated to achieve a self-consistent solution for the observed gas expansion velocity (Lombaert et al. 2012). Recently, De Beck et al. (2010) found $\dot{M}_{\mathrm{g}}=3.1 \times 10^{-5} M_{\odot} \mathrm{yr}^{-1}$ for LL Peg, but indicated a possible mass-loss variability. We as- 
Table 1. Chemical formula, specific density $\rho_{\mathrm{s}}$, condensation temperature $T_{\text {cond }}$, and condensation radius $R_{\text {cond }}$ of the dust species.

\begin{tabular}{lrrrrr}
\hline \hline Dust species & $\begin{array}{r}\text { Chem. } \\
\text { form. }\end{array}$ & $\begin{array}{r}\rho_{\mathrm{s}} \\
\left(\mathrm{g} \mathrm{cm}^{-} 3\right)\end{array}$ & $\begin{array}{r}T_{\text {cond }} \\
(\mathrm{K})\end{array}$ & $\begin{array}{r}R_{\text {cond }} \\
\left(R_{\star}\right)\end{array}$ & Ref. \\
\hline Amorphous carbon & $\mathrm{a}-\mathrm{C}$ & 1.8 & 1650 & 2.5 & 1 \\
Metallic iron & $\mathrm{Fe}$ & 7.9 & 1100 & 7.0 & 2 \\
Silicon carbide & $\mathrm{SiC}$ & 3.2 & 1400 & 4.0 & 3 \\
Magnesium sulfide & $\mathrm{MgS}$ & 3.0 & 750 & 11.0 & 4 \\
\hline
\end{tabular}

Notes. The final column lists the reference to the optical constants: 1). Jäger et al. (1998); 2). Henning \& Stognienko (1996); 3). Pitman et al. (2008); 4). Begemann et al. (1994).

sumed $\dot{M}_{\mathrm{g}}=3 \times 10^{-5} M_{\odot} \mathrm{yr}^{-1}$, which leads to a dust expansion velocity of $16 \mathrm{~km} \mathrm{~s}^{-1}$. The stellar effective temperature of LL Peg was taken to be $T_{\star}=2400 \mathrm{~K}$. The adopted temperature does not impact the results because the CSE of LL Peg is optically thick up to $\lambda \sim 50 \mu \mathrm{m}$. We used a luminosity of $L_{\star}=1.1 \times 10^{4} L_{\odot}$ at a distance of $d_{\star}=1300 \mathrm{pc}$, following the period-luminosity relation of Groenewegen \& Whitelock (1996). The stellar radius is thus $R_{\star}=608 R_{\odot}$.

Table 1 lists the dust species used in this study and relevant dust properties. The bulk of the dust composition is provided by amorphous carbon (a-C). We included a small fraction of metallic iron (Fe), which is a common dust component in the outflow of carbon stars (Lattimer et al. 1978). We used silicon carbide ( $\mathrm{SiC}$ ) to model the $11 \mu \mathrm{m}$ feature (see Speck et al. 2009, and references therein for a more detailed discussion on $\mathrm{SiC}$ as a dust component in carbon-rich envelopes), and $\mathrm{MgS}$ to model the $30 \mu \mathrm{m}$ feature (see Sect. 4). The optical properties of these dust species were derived from reflection measurements in the laboratory. References for these measurements are listed in Table 1. We used three models to represent grain shapes (Bohren \& Huffman 1983; Min et al. 2003): a continuous distribution of ellipsoids (CDE), a distribution of hollow spheres with filling factor 0.8 (DHS), and spherical particles (MIE). We adopted a single grain size of $0.01 \mu \mathrm{m}$ for all grain shape models.

In constructing the optical properties of composite grains we summed up the extinction contributions of the separate dust species. This is a valid assumption if absorption and emission occur in the Rayleigh limit, i.e. at $\lambda \gg 0.01 \mu \mathrm{m}$ (Min et al. 2008). Moreover, the refractive indices of $\mathrm{MgS}$ are significantly higher in the $30 \mu \mathrm{m}$ feature than those of the other dust species included in the model and, for the relative abundances found in this study, dominate the spectral behavior of the composite grains in this wavelength region. This supports our approach. Alternatively, one may compute the optical properties of composite grains. This would require assuming a composite structure, which may add a larger uncertainty to the spectral shape than the assumption of summing the individual extinction contributions.

The $11 \mu \mathrm{m}$ feature is best fitted with $\mathrm{SiC}$ dust in a CDE ensemble. Using the $\mathrm{CDE}$ ensemble for a-C grains, however, significantly increases the emission at $\lambda>50 \mu \mathrm{m}$, as shown by the full green curve in Fig. 1. The full blue model, on the other hand, adopts the DHS particle shapes for a-C, yielding better results. We find a dust-mass-loss rate of $\dot{M}_{\mathrm{d}}=(1.7 \pm 0.1) \times 10^{-7} M_{\odot} \mathrm{yr}^{-1}$, comprised of $70 \% \mathrm{a}-\mathrm{C}, 5 \% \mathrm{Fe}, 10 \% \mathrm{SiC}$ and $15 \% \mathrm{MgS}$.

\section{The $\mathbf{3 0} \mu \mathrm{m}$ feature: resolving the mass problem}

In principle, the result of a model including MgS may critically depend on the optical constants assumed for $\mathrm{MgS}$ at $\lambda<10 \mu \mathrm{m}$, since the shape of the $30 \mu \mathrm{m}$ feature is very sensitive to the temperature distribution of the $\mathrm{MgS}$ grains (Hony et al. 2002).

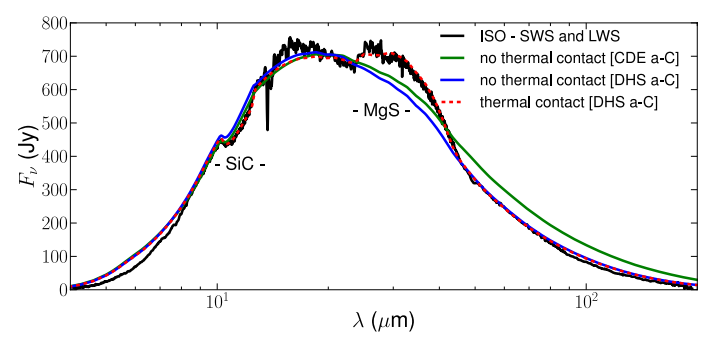

Fig. 1. SED of LL Peg. The SWS and LWS data are shown in full black. The dashed red curve shows a model assuming thermal contact between all dust species, whereas the full blue line shows a model assuming no thermal contact. The dust composition of both models consists of a-C grains in DHS shapes and $\mathrm{SiC}, \mathrm{MgS}$ and $\mathrm{Fe}$ grains in $\mathrm{CDE}$ shapes. The full green model includes a-C grains in CDE shapes and assumes no thermal contact.

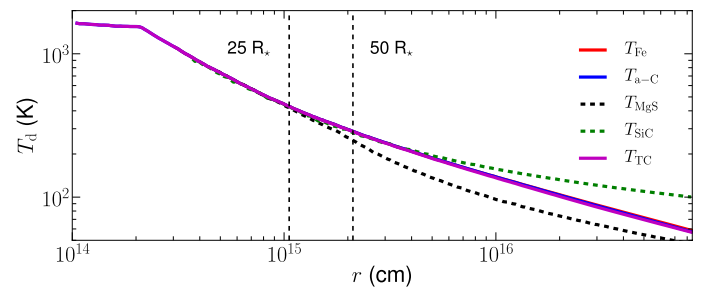

Fig. 2. Dust temperature profile of the envelope of LL Peg for individual species and composite grains: $\mathrm{Fe}$ in red, a-C in blue, $\mathrm{MgS}$ in dashedblack, $\mathrm{SiC}$ in dashed-green and composite grains in magenta. Note that the red, blue and magenta profiles essentially coincide throughout the whole envelope.

For stars that have a low mass-loss rate this introduces a large uncertainty in the spectral behavior of $\mathrm{MgS}$ because the heating of this species is caused by direct stellar light at precisely these wavelengths. However, for a high mass-loss-rate star such as LL Peg, the model dependence on the unknown short wavelength MgS optical constants disappears. The wind of LL Peg is optically thick up to $\lambda \sim 50 \mu \mathrm{m}$ with the transition from an optically thick to an optically thin envelope in the infrared occurring between 15 and $35 R_{\star}$. For individual species the dust temperature profiles coincide in the optically thick region of the outflow, see Fig. 2. In the optically thin, outer wind the $\mathrm{MgS}$ and $\mathrm{SiC}$ profiles start to deviate from those of a-C and Fe. The $30 \mu \mathrm{m}$ feature is produced by emission from dust particles in the outer envelope, which are heated by the infrared radiation emitted from an optically thick surface at $\sim 25 R_{\star}$. The dust temperature at this radial distance is $\sim 400 \mathrm{~K}$, such that most of the heating occurs at $\lambda>7 \mu \mathrm{m}$.

As indicated by Zhang et al. (2009), the strength of the $30 \mu \mathrm{m}$ feature in HD 56126 cannot be reproduced using pure $\mathrm{MgS}$ grains, unless the $\mathrm{MgS}$ abundance far exceeds the amount of atomic sulfur that is available from atmospheric abundance estimates. This mass problem is shown in the full blue model in Fig. 1, which assumes an amount of MgS dust in agreement with the solar abundance of sulfur. However, Zhang et al. used chemically homogeneous grains, which heat and cool independently of grains of a different chemical composition. If all dust species are in thermal contact, the temperature distribution (shown by the magenta curve in Fig. 2) is predominantly set by the chemical component that is both reasonably abundant and is an efficient absorber. For a carbon-rich environment, a-C grains heat and cool more efficiently over a broader wavelength range than $\mathrm{MgS}$ or SiC grains. As shown by the dashed red curve in Fig. 1, assuming thermal contact between the dust species, but otherwise identical parameters as in the blue model, indeed results in a $30 \mu \mathrm{m}$ feature that is produced well in both strength and shape. 
We quantified our results by calculating the atomic number abundance with respect to $\mathrm{H}_{2}$ of sulfur needed for the $\mathrm{MgS}$ dust mass in our models. We only considered sulfur, since it is the least abundant component with a solar atomic abundance of $(2.6 \pm 0.2) \times 10^{-5}$ (Asplund et al. 2009). Two models were calculated, one assuming thermal contact between dust species (see the dashed red curve in Fig. 1), and one assuming no thermal contact (see the dashed blue curve in Fig. 3 for the continuumdivided $30 \mu \mathrm{m}$ feature). In both models, the dust composition and dust-mass-loss rate were adapted such that the $30 \mu \mathrm{m}$ feature is reproduced with a similar equivalent width. The model assuming thermal contact yields an atomic number abundance of $2.5 \times 10^{-5}$ for sulfur. The model without thermal contact requires an abundance of $1.2 \times 10^{-4}$. Given the potentially variable mass loss of LL Peg (e.g. Mauron \& Huggins 2006), we estimate the uncertainty on these values to be a factor of two. In the case of thermal contact, the required amount of $\mathrm{MgS}$ to reproduce the $30 \mu \mathrm{m}$ feature is within the limits imposed by a solar sulfur abundance, independent of the particle shape model. Without assuming thermal contact, the required amount of $\mathrm{MgS}$ would significantly exceed a solar atomic sulfur abundance.

\section{Discussion}

\subsection{Homogeneous versus composite grains}

The above motivated need for thermal contact between the dust species implies that they must be included in some kind of heterogeneous composite grain structure. Recently, Zhukovska \& Gail (2008) discussed the possibility of forming MgS dust in carbonaceous environments through precipitation on $\mathrm{SiC}$ precursor grains, which would be a plausible way to achieve composite grains. These authors showed that the formation of $\mathrm{MgS}$ is strongly coupled to that of $\mathrm{SiC}$, a process in which sulfur is being freed by breaking up SiS molecules. This sulfur is stored in $\mathrm{H}_{2} \mathrm{~S}$, which can react with freely available magnesium to condense into $\mathrm{MgS}$. A connection between molecular SiS and the formation of $\mathrm{MgS}$ is also supported by Smolders et al. (2012), who found a strong correlation between the presence of SiS molecular bands and a $30 \mu \mathrm{m}$ feature in their sample of S-stars. SiC is not formed in these stars, but $\mathrm{SiS}$ can react directly with $\mathrm{Mg}$ to form $\mathrm{MgS}$, albeit less efficiently. This scenario agrees with the significantly lower $\mathrm{MgS}$ abundance that is needed to explain the typically weak $30 \mu \mathrm{m}$ feature in S-stars.

Additional support for the above hypothesis was given by Leisenring et al. (2008), who compared galactic carbon stars with a sample in the LMC, in which all sources show an $\mathrm{SiC}$ feature at $11 \mu \mathrm{m}$, while only half of the sources show emission at $30 \mu \mathrm{m}$. Stronger $30 \mu \mathrm{m}$ features are found together with weaker $\mathrm{SiC}$ features, which led Leisenring and collaborators to suggest that $\mathrm{MgS}$ forms as a coating on top of $\mathrm{SiC}$ grains. In galactic AGB stars, a-C is expected to form first, followed by $\mathrm{SiC}$. If it is energetically beneficial for $\mathrm{SiC}$ to form on top of a-C grains in a core-mantle structure, or for homogenous a-C and $\mathrm{SiC}$ grains to stick together in an aggregate structure, it is likely that composite grains are formed. For core-mantle grains, Zhukovska \& Gail (2008) showed that a resonance effect caused by such a structure induces a second peak at $\sim 35 \mu \mathrm{m}$ in the absorption efficiency profile of spherical MgS grains. However, an ensemble of nonspherical grains will likely not produce such a resonance effect, see Sect. 5.2.

$\mathrm{MgS}$ formation directly from the gas-phase - without any kind of precursor grain of different composition - would provide a mechanism to produce chemically homogeneous grains. However, several studies have indicated that this process is

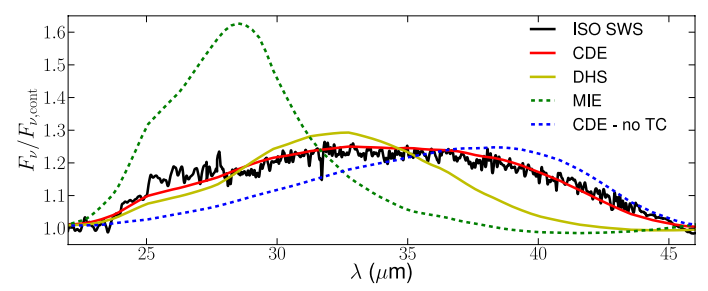

Fig. 3. Continuum-divided $30 \mu \mathrm{m}$ feature with the SWS data of LL Peg shown in full black. The CDE model in full red assumes thermal contact and requires a sulfur abundance of about the solar value. In the model represented by the dashed blue line the CDE particles are not in thermal contact. To fit the strength of the feature sulfur needs to be $\sim 5$ times the solar value. A DHS (full yellow line) and MIE (dashed green) model represent alternative shape distributions of particles in which the dust components are in thermal contact. The sulfur abundance in the MIE model has been adjusted to match the equivalent width of the observed feature.

not sufficiently efficient to explain the large amounts of $\mathrm{MgS}$ needed to produce a strong $30 \mu \mathrm{m}$ feature (Kimura et al. 2005; Zhukovska \& Gail 2008; Cherchneff 2012). In their study of carbon-rich AGB stars and planetary nebulae, Hony et al. (2002) adopted chemically homogeneous grains and derived a temperature for the $\mathrm{MgS}$ particles by fitting the $30 \mu \mathrm{m}$ feature after subtraction of a smooth continuum. For LL Peg, they found a continuum (i.e. essentially a-C) temperature of $340 \mathrm{~K}$ and a $\mathrm{MgS}$ temperature of $120 \mathrm{~K}$. They ascribed the difference to the chemical homogeneity of the grains. However, the authors assumed that MgS is formed in an optically thin medium and can be characterized by a unique temperature. Our radiative transfer calculations show that both these assumptions are not valid for LL Peg. Moreover, the low temperature of $\mathrm{MgS}$ found by Hony et al. leads to the MgS mass problem. We conclude that a population of chemically homogeneous grains, and hence no thermal contact between dust species, cannot be reconciled with the spectrum of LL Peg.

\subsection{Particle shape and size}

The spectral shape of a dust emission feature may strongly depend on the model that is used to describe the particle shapes (Hony et al. 2002; Mutschke et al. 2009). The continuum-divided $\mathrm{MgS}$ features for several particle models are shown in Fig. 3. Mie particles clearly cannot reproduce the spectral profile around $30 \mu \mathrm{m}$ in LL Peg. Several studies (e.g. Hony et al. 2002; Min et al. 2003) have indicated that Mie particles in any grain-size distribution cause narrow features and certain resonances, which have not been observed in the thermal continuum emission of AGB stars. It is generally better to use an ensemble of particle shapes, such as given by a CDE or DHS (see Mutschke et al. 2009 for an overview of these and more advanced particle shape models), because they more accurately represent features caused by a collection of irregularly shaped dust particles. Note that for both the CDE and the DHS model the extinction properties are independent of our single grain-size assumption as long as the Rayleigh limit holds. The assumption of homogeneous or composite grains also impacts the shape of the $\mathrm{MgS}$ feature, because the dust temperature profile is affected. If one assumes homogeneous grains (i.e. no thermal contact) and increases the $\mathrm{MgS}$-dust mass such that the strength of the feature is approximated, one finds that the $30 \mu \mathrm{m}$ feature shape is rather poorly reproduced by a CDE model (see the dashed blue curve in Fig. 3). However, this poor agreement cannot be used as an argument for the need of thermal contact because CDE is quite a simple grain shape model. A comparative study between particle shape 


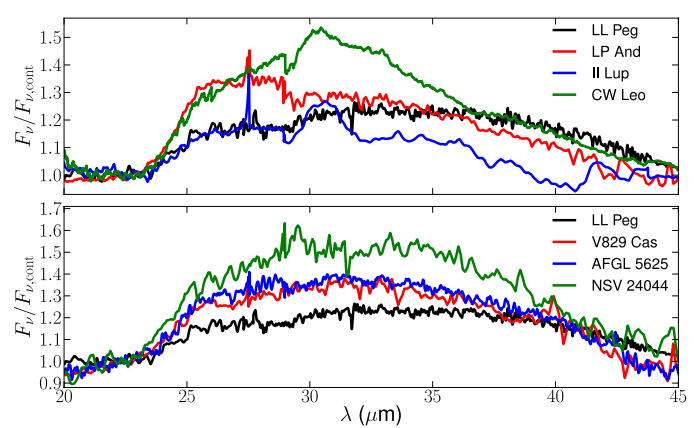

Fig. 4. Continuum-divided ISO spectra of multiple carbon stars in the region of the $30 \mu \mathrm{m}$ feature, with LL Peg as a reference in black. The top panel shows three typical AGB stars with relatively low-density envelopes; the bottom panel shows three typical AGB stars with relatively high density-envelopes (data taken from Hony et al. 2002).

models by Mutschke et al. (2009) has shown that the CDE model often results in emission bands too much enhanced at red wavelengths when compared to dust optical properties measured in the laboratory. The good fit of our MgS CDE model given by the red curve and the observed $30 \mu \mathrm{m}$ feature in LL Peg consequently may indicate some additional effect that enhances the red wing of the observed band profile. Condensation experiments by Kimura et al. (2005) have shown that MgS dust formed as network-like structures can show an enhanced red part of the MgS band profile. Spectra of dust grains embedded in a matrix also show such an enhancement compared to those of free particles (e.g. Tamanai et al. 2006), which may point to mixing or embedding of the MgS dust in inhomogeneous grains.

\subsection{Diversity of the $30 \mu \mathrm{m}$ feature shape in AGB outflows}

LL Peg is a highly evolved AGB star and exhibits one of the reddest thermal emission continua of all carbon stars. The outflow of this source is optically thick even in the $30 \mu \mathrm{m}$ feature. As indicated in the bottom panel of Fig. 4, AGB stars with a high-density envelope show an almost uniform shape of the $30 \mu \mathrm{m}$ feature. Sources with a lower density envelope, such as those shown in the top panel of Fig. 4, display a wide diversity in shape. As a first possible explanation of this phenomenon, Kimura et al. (2005) suggested that the shape of the $30 \mu \mathrm{m}$ feature may depend on the formation history of $\mathrm{MgS}$. If the grains have formed through gas-phase condensation, the resulting emission feature is expected to be more pronounced at $\lambda \sim 32 \mu \mathrm{m}$ than at longer wavelengths. If $\mathrm{MgS}$ grains are produced via a gas-solid reaction, the red wing of the feature at $\lambda>35 \mu \mathrm{m}$ becomes more intense relative to the strength at $\lambda \sim 32 \mu \mathrm{m}$. As shown in Fig. 4, the $30 \mu \mathrm{m}$ feature in the high-density envelopes has a more pronounced red wing than those in the lower density envelopes. One may speculate that in low-density sources, where the $30 \mu \mathrm{m}$ feature is optically thin throughout the entire envelope, the feature may be composed of gas-phase condensates in the inner part of the wind and grains formed by gas-solid reactions throughout or in the outer part of the wind. In high-density sources, one only observes the contributions from the latter formation channel. This scenario could be consistent with the MgS-SiC correlation in S-type stars (Smolders et al. 2012), where the lack of SiC particles indicates a gas-phase condensation channel, albeit not a very effective one. A second explanation might be that the low opacities in low-density envelopes favor the heating of another dust species with an absorption coefficient profile peaking at about $30 \mu \mathrm{m}$. Alternative carriers suggested in the literature include hydrogenated amorphous carbons (Grishko et al. 2001).

L18, page 4 of 4

\section{Conclusions}

We have modeled the ISO spectrum of the high-density carbon star LL Peg with a dust composition consisting of a-C, Fe, SiC, and $\mathrm{MgS}$. The (high) density and temperature structure in the envelope of this source allow one to model the $30 \mu \mathrm{m}$ feature with $\mathrm{MgS}$ dust particles, independent of the unknown $\mathrm{MgS}$ optical properties at $\lambda<10 \mu \mathrm{m}$. We showed that $\mathrm{MgS}$ is a viable candidate to be the carrier of the $30 \mu \mathrm{m}$ feature. An ensemble of particle shapes works significantly better than spherical grains to explain the shape of the feature. Thermal contact between the dust species is required to ensure that the amount of $\mathrm{MgS}$ dust in the envelope of LL Peg does not exceed the solar abundance of sulfur, thereby avoiding the mass problem as reported by Zhang et al. (2009) for the post-AGB star HD 56126. Achieving thermal contact between all dust species is possible if these species form in some kind of heterogeneous composite grain structure.

Acknowledgements. We thank S. Hony for providing the reduced SWS spectra of the high-density carbon stars. We also express gratitude toward the referee, who provided instructive feedback. R.L. acknowledges financial support from the Fund for Scientific Research - Flanders (FWO) under grant number ZKB5757-04-W01, and from the Department of Physics and Astronomy of the KULeuven. BdV and KS acknowledge financial support from the FWO.

\section{References}

Asplund, M., Grevesse, N., Sauval, A. J., \& Scott, P. 2009, ARA\&A, 47, 481 Begemann, B., Dorschner, J., Henning, T., Mutschke, H., \& Thamm, E. 1994, ApJ, 423, L71

Bjorkman, J. E., \& Wood, K. 2001, ApJ, 554, 615

Bohren, C. F., \& Huffman, D. R. 1983, Absorption and scattering of light by small particles (New York: Wiley)

Cherchneff, I. 2012, A\&A, in press, DOI: 10.1051/0004-6361/201118542

Chiar, J. E., \& Tielens, A. G. G. M. 2006, ApJ, 637, 774

De Beck, E., Decin, L., de Koter, A., et al. 2010, A\&A, 523, A18

Decin, L., Hony, S., de Koter, A., et al. 2006, A\&A, 456, 549

Decin, L., De Beck, E., Brünken, S., et al. 2010, A\&A, 516, A69

Drimmel, R., Cabrera-Lavers, A., \& López-Corredoira, M. 2003, A\&A, 409, 205

Goebel, J. H., \& Moseley, S. H. 1985, ApJ, 290, L35

Grishko, V. I., Tereszchuk, K., Duley, W. W., \& Bernath, P. 2001, ApJ, 558, L129

Groenewegen, M. A. T., \& Whitelock, P. A. 1996, MNRAS, 281, 1347

Henning, T., \& Stognienko, R. 1996, A\&A, 311, 291

Hony, S., Waters, L. B. F. M., \& Tielens, A. G. G. M. 2002, A\&A, 390, 533

Hrivnak, B. J., Volk, K., \& Kwok, S. 2000, ApJ, 535, 275

Jäger, C., Mutschke, H., \& Henning, T. 1998, A\&A, 332, 291

Jiang, B. W., Szczerba, R., \& Deguchi, S. 1999, A\&A, 344, 918

Kessler, M. F., Steinz, J. A., Anderegg, M. E., et al. 1996, A\&A, 315, L27

Kimura, Y., Kurumada, M., Tamura, K., et al. 2005, A\&A, 442, 507

Lattimer, J. M., Schramm, D. N., \& Grossman, L. 1978, ApJ, 219, 230

Leisenring, J. M., Kemper, F., \& Sloan, G. C. 2008, ApJ, 681, 1557

Lombaert, R., Decin, L., de Koter, A., et al. 2012, A\&A, submitted

Mauron, N., \& Huggins, P. J. 2006, A\&A, 452, 257

Min, M., Hovenier, J. W., \& de Koter, A. 2003, A\&A, 404, 35

Min, M., Hovenier, J. W., Waters, L. B. F. M., \& de Koter, A. 2008, A\&A, 489, 135

Min, M., Dullemond, C. P., Dominik, C., de Koter, A., \& Hovenier, J. W. 2009, A\&A, 497, 155

Mutschke, H., Min, M., \& Tamanai, A. 2009, A\&A, 504, 875

Pitman, K. M., Hofmeister, A. M., Corman, A. B., \& Speck, A. K. 2008, A\&A, 483,661

Sloan, G. C., Kraemer, K. E., Price, S. D., \& Shipman, R. F. 2003, ApJS, 147, 379

Smolders, K., Neyskens, P., Blommaert, J. A. D. L., et al. 2012, A\&A, 540, A72 Speck, A. K., Corman, A. B., Wakeman, K., Wheeler, C. H., \& Thompson, G. 2009, ApJ, 691, 1202

Szczerba, R., Henning, T., Volk, K., Kwok, S., \& Cox, P. 1999, A\&A, 345, L39

Tamanai, A., Mutschke, H., Blum, J., \& Meeus, G. 2006, ApJ, 648, L147

Volk, K., Kwok, S., Hrivnak, B. J., \& Szczerba, R. 2002, ApJ, 567, 412

Yamamura, I., de Jong, T., Justtanont, K., Cami, J., \& Waters, L. 1998, Ap\&SS, 255,351

Zhang, K., Jiang, B. W., \& Li, A. 2009, ApJ, 702, 680

Zhukovska, S., \& Gail, H.-P. 2008, A\&A, 486, 229 